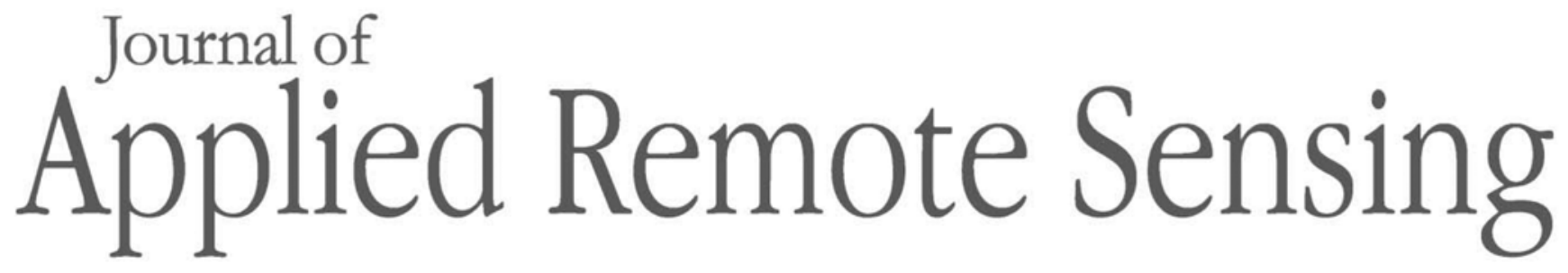

RemoteSensing.SPIEDigitalLibrary.org

\title{
Extension of the constrained ratio approach to aerosol retrievals from elastic-scatter and high spectral resolution lidars
}

Christopher J. McPherson John A. Reagan 


\title{
Extension of the constrained ratio approach to aerosol retrievals from elastic-scatter and high spectral resolution lidars
}

\author{
Christopher J. McPherson ${ }^{\mathrm{a}, *}$ and John A. Reagan ${ }^{\mathrm{b}}$ \\ ${ }^{a}$ Arizona Optical Systems, LLC, 5575 South Houghton Road, Tucson, \\ Arizona 85747, United States \\ ${ }^{\mathrm{b}}$ University of Arizona, Department of Electrical and Computer Engineering, \\ 1230 East Speedway Boulevard, Tucson, Arizona 85721, United States
}

\begin{abstract}
A methodology is presented, by which atmospheric aerosol retrievals from a standard, elastic-scatter, lidar can be constrained by using information from coincident measurements from a high spectral resolution lidar (HSRL) or Raman lidar at a different wavelength. As high spectral resolution or inelastic-scattering lidars are now being incorporated coaxially into instruments with traditional, elastic-scatter channels at different wavelengths, a standard approach is needed to incorporate or fuse the diversity of spectral information so as to make maximal use of the aerosol measurements made from the elastic-scatter channel or channels. The approach is evaluated through simulation and with data from the NASA Langley Research Center Airborne HSRL instrument. The generality and extensibility of the method is also explored and discussed in the context of aerosol modeling. (C) The Authors. Published by SPIE under a Creative Commons Attribution 3.0 Unported License. Distribution or reproduction of this work in whole or in part requires full attribution of the original publication, including its DOI. [DOI: 10.1117/1.JRS.10.036019]
\end{abstract}

Keywords: lidar; aerosols; algorithms.

Paper 16260 received Apr. 10, 2016; accepted for publication Aug. 4, 2016; published online Aug. 23, 2016.

\section{Introduction}

The problem of atmospheric aerosol retrieval from elastic-scatter lidar measurements has been approached in a number of different ways, each incorporating or imposing various assumptions and constraints to the underdetermined nature of the problem. Frequently, the most appropriate approach for a given dataset depends upon what additional information may be available to narrow the solution space to an appropriate degree to facilitate a solution in a given context. In all cases, some relationship exists between the more-or-less spatially and temporally independent processes of aerosol backscatter and extinction. The process of aerosol "retrieval" necessarily consists of predicting or estimating this relationship in a manner suitable to whatever external information may exist. Typically, the relationship between aerosol backscatter and extinction is expressed in terms of a ratio, the so-called "lidar ratio," signifying its importance in the solution. Given the all-important lidar ratio, various mathematical solutions exist to the underlying differential equation to determine the aerosol backscatter and extinction based upon different boundary constraints that may apply in different contexts. ${ }^{1-4}$ Whatever solution may be applied to aerosol retrievals, the goal is ultimately the accurate estimation of the lidar ratio, which does of course vary spectrally, spatially, and temporally based upon the several flavors of varying properties of the aerosol in the atmosphere. Various constraints are usually applied to the lidar ratio (or to other relevant ratios) to facilitate a solution. For example, independent measurements of aerosol spectral optical depth (or surface reflectance) can be used to complement lidar measurements and constrain the lidar solution to be consistent with the measured optical depth (or that implied by a surface reflectance constraint), in the context of an assumed spatially fixed lidar ratio at a given wavelength. ${ }^{5}$

*Address all correspondence to: Christopher J. McPherson, E-mail: cjm3@email.arizona.edu 
One technique known as the constrained ratio aerosol model-fit (CRAM), developed originally to facilitate aerosol retrievals from spaceborne multiwavelength lidars, attempts to impose the constraint of a set of aerosol models to reach a solution at two wavelengths that is consistent (in terms of certain spectral ratio intensive properties) with one of several aerosol model types developed from extensive global measurements. ${ }^{6,7}$ Considerable analysis of accumulated sunphotometer data from the Aerosol Robotic Network (AERONET) ${ }^{8}$ described a fairly comprehensive global aerosol climatology, resulting in models characterizing five key aerosol types in terms of lidar ratios at 550 and $1020 \mathrm{~nm}$ as well as spectral ratios of aerosol extinction and backscatter at these wavelengths. ${ }^{9}$ These aerosol models formed the basis for CRAM, with the understanding that the wavelengths were close enough to the Nd:YAG wavelengths on instruments like the Geoscience Laser Altimeter System and the Cloud-Aerosol Lidar with Orthogonal Polarization (CALIOP) lidar aboard the Cloud-Aerosol Lidar and Infrared Pathfinder Satellite Observations (CALIPSO) satellite to be useful for the purposes of constraining retrieval solutions from these instruments. The CRAM technique has proven useful for aerosol retrievals from CALIPSO, at least in situations in which the observed aerosol turns out to be consistent with one of the models. ${ }^{10}$

As science missions develop to study atmospheric aerosols, different combinations of instruments or of measurement techniques lend themselves to new and promising approaches to the problem of retrievals. Increasingly, inelastic-scattering instruments such as Raman lidars ${ }^{11,12}$ or instead, instruments such as high spectral resolution lidar (HSRL) are being deployed coaxially with conventional elastic-scatter channels at different wavelengths in a unified system, ${ }^{13}$ often coupling or otherwise simplifying the calibration of the various channels in such a way as to provide data of different types that are temporally and spatially coincident.

The NASA Langley Research Center (LaRC) Airborne HSRL ${ }^{13}$ incorporates a high spectral resolution, constantly tuned 532-nm channel together with a conventional 1064-nm elasticscattering channel into a robust, reliable airborne system that has to date flown many hundreds of hours across the North American continent, offshore, and over the Caribbean, in numerous measurement campaigns, accompanied at times by a host of validating sensors. ${ }^{14-20}$ We propose a retrieval scheme, basically an extension or modification of the CRAM technique (called E-CRAM), that attempts to take advantage of the added information available from HSRL at $532 \mathrm{~nm}$ in the dual-wavelength retrieval. ${ }^{21}$ Since range-resolved profiles of aerosol backscatter and extinction are available at $532 \mathrm{~nm}$ via HSRL, the retrieval component of E-CRAM applies just to the 1064-nm elastic-scatter data. The retrieval at $1064 \mathrm{~nm}$ benefits from the aerosol information at $532 \mathrm{~nm}$, in particular the backscatter profile, which is essentially proportional to the vertical aerosol loading profile (if the aerosol layer is assumed to have spatially uniform intensive properties), which is then used to constrain the solution at $1064 \mathrm{~nm}$. The technique is conceptually somewhat similar to the dual-wavelength retrieval approach taken by Sasano and Browell, ${ }^{22}$ except that the 532-nm aerosol extinction and backscatter profiles are known, thus reducing the number of degrees of freedom in the retrieval to one from two, resulting in more stable and reliable solutions.

\section{Retrieval Method}

The retrieval approach described can be applied to aerosol measurements by an HSRL-type instrument. The constraint of spatial uniformity refers only to the optical and microphysical (intensive, rather than extensive) properties of the aerosol. With the imposition of this constraint, one is essentially limiting the spectral behavior of the aerosol backscatter and lidar ratio to a fixed spatial relationship. That is, that the single scatter albedo and phase function, the properties that determine the lidar ratio and are themselves functions of physical properties of the aerosol, i.e., complex refractive index and particle size distribution, remain fairly constant spatially. In this case, a fixed spatial relationship is assumed to exist between the spectral lidar and aerosol backscatter ratios. In general, this is always true of aerosol at a given point, so the constraint applied by the technique assumes only that this behavior remains relatively constant spatially. Thus, the lidar ratio at $1064 \mathrm{~nm}$ can be expressed as a fixed multiple of the lidar ratio at $532 \mathrm{~nm}$, i.e., 


$$
S_{a, 1064}=K_{1} S_{a, 532},
$$

where $K_{1}$ is a fixed value in some region in which the aerosol is assumed to be homogeneous and not strongly spatially variant. Similarly, the aerosol backscatter at $1064 \mathrm{~nm}$ is expressed as a fixed multiple of the backscatter at $532 \mathrm{~nm}$ via a second fixed multiple, i.e.,

$$
\beta_{a, 1064}=K_{2} \beta_{a, 532} \text {. }
$$

The expression for the aerosol extinction at $1064 \mathrm{~nm}$ can also be given in terms of $K_{1}, K_{2}$ and the aerosol extinction at $532 \mathrm{~nm}$

$$
\sigma_{a, 1064}=K_{1} K_{2} \sigma_{a, 532}
$$

Although there appear to still be two degrees of freedom in $K_{1}$ and $K_{2}$, the terms are coupled by the retrieval process, since a choice of $K_{1}$ determines the 1064-nm lidar ratio, which in turn determines the aerosol backscatter and extinction at $1064 \mathrm{~nm}$. Since the aerosol backscatter and extinction are available at $532 \mathrm{~nm}$ via HSRL, $K_{2}$ is dependent upon the choice of $K_{1}$. The solution according to E-CRAM attempts to choose $K_{1}$ such that the spatial variability in $K_{2}$ is minimized in some region of homogeneous aerosol. Importantly, the aerosol loading need not be spatially homogeneous, only the microphysical properties governing the spectral ratio parameters, i.e., intensive rather than extensive properties of the aerosol. In addition, the relative degree of spatial homogeneity of the aerosol intensive microphsyical properties can be assessed fairly directly via the lidar ratio at $532 \mathrm{~nm}$ available via HSRL measurements.

Different choices of techniques are available for the actual retrieval of aerosol extinction and backscatter from elastic-scatter lidar measurements given a value of the lidar ratio. Two of the more common are attributable to Klett ${ }^{3,4}$ and Fernald. ${ }^{1,2}$ The solution according to Fernald meets the requirements of E-CRAM rather well, as it is based upon a transmittance boundary constraint that is easy to determine in regions near an airborne lidar. For a single-wavelength solution in a two-component atmosphere, i.e., independent aerosol and Rayleigh scattering, the Fernald backscatter retrieval equation is given by

$$
\beta_{a}(r)=\frac{X(r) \exp \left\{-2 \int_{r_{0}}^{r}\left[S_{a}\left(r^{\prime}\right)-S_{R}\right] \beta_{R}\left(r^{\prime}\right) \mathrm{d} r^{\prime}\right\}}{T^{2}\left(r_{0}\right)-2 \int_{r_{0}}^{r} S_{a}\left(r^{\prime}\right) X\left(r^{\prime}\right) \exp \left\{-2 \int_{r_{0}}^{r^{\prime}}\left[S_{a}\left(r^{\prime \prime}\right)-S_{R}\right] \beta_{R}\left(r^{\prime \prime}\right) \mathrm{d} r^{\prime \prime}\right\} \mathrm{d} r^{\prime}} .
$$

The $X(r)$ term refers to the attenuated backscatter that, for a calibrated elastic-scatter lidar, is equivalent to the product of the total backscatter at range $r$ and the round-trip transmittance to and from range $r$ (the squared exponential of the integrated total extinction). Terms with subscript $R$ refer to the Rayleigh scattering components, which can be predicted very accurately with knowledge of the atmospheric temperature and pressure profiles. The $T^{2}\left(r_{0}\right)$ term denotes the round-trip transmittance between the lidar and the point $r_{0}$ at which the forward retrieval starts.

Spatial regions suitable for E-CRAM analysis can be identified by placing certain constraints on the spatial variability of the lidar ratio at $532 \mathrm{~nm}$, which is one of the calibrated data products of the LaRC Airborne HSRL. Examples with simulated data will demonstrate that E-CRAM solutions are readily obtainable for situations in which the spatial variability of the 532-nm lidar ratio is characterized by normally distributed random variation with standard deviation relative to a mean value of at most $\sim 15 \%$. In practice with HSRL data, regions are identified such that the standard deviation of the measured 532-nm lidar ratio is within $15 \%$ of a mean value, with no clear spatial trends. This has generally been the adopted requirement for spatial homogeneity of the aerosol within an E-CRAM solution region. Once a suitable retrieval region has been identified in the data (with no significant aerosol layer above), an iterative solution on $K_{1}$ can be applied to find the appropriate ratio of the 1064-nm lidar ratio (relative to $532 \mathrm{~nm}$ ) to minimize the spatial variability on the spectral aerosol backscatter ratio, i.e., finding $K_{1}$ which minimizes the spatial variability of $K_{2}$. The spatial variability on the retrieved $K_{2}$ ratio quantity is assessed in terms of the spatial coefficient of variation, i.e., the standard deviation relative to the mean or, more colloquially, "sigma-over-mu." 


\section{Proof-of-Concept Simulations}

A number of proof-of-concept simulations were undertaken to test the suitability and sensitivity of E-CRAM to aerosol layers in which the 532-nm lidar ratio was not perfectly invariant spatially, and to the presence of noise in the data as well as errors in the calibration at $1064 \mathrm{~nm}$. The E-CRAM approach carries two principal sensitivities, to the calibration of the 1064-nm attenuated backscatter channel and to the 532-nm lidar ratio measurement. These error terms may in practice be individually spatially correlated (as with a bias) or spatially uncorrelated due to random signal or measurement error. Although extensive in-flight calibration is undertaken to ensure minimal bias error in the measurements, it is important to understand the sensitivity of the technique to spatially correlated error due to the spatial nonlinearity of the retrieval. Fortunately, the algorithm is not particularly sensitive to these nonlinearities since the retrieval of the 1064-nm lidar ratio depends upon a spectral ratio quantity, specifically the spectral aerosol backscatter ratio. Mock aerosol extinction and backscatter profiles were created at $532 \mathrm{~nm}$, assuming parameters consistent with the mean values of one of the CRAM models, in this case the Southeast Asian aerosol model, having a 532-nm (actually $550 \mathrm{~nm}$ ) lidar ratio of $58 \pm 10 \mathrm{sr}$, a 1064-nm (actually $1020 \mathrm{~nm}$ ) lidar ratio of $38.7 \pm 6.7 \mathrm{sr}$ (for a $550-/ 1020-\mathrm{nm}$ spectral ratio of the lidar ratio of 1.5 ), spectral backscatter ratio (550 to $1020 \mathrm{~nm}$ ) of $1.6 \pm 0.2$, and a spectral extinction ratio (550 to $1020 \mathrm{~nm}$ ) of $2.4 \pm 0.3$. With an attenuated backscatter profile at $1064 \mathrm{~nm}$ created using a measured Rayleigh scattering profile from HSRL data, together with the backscatter and extinction profiles created at $532 \mathrm{~nm}$, the ability of E-CRAM to correctly determine the $K_{1}$ parameter for the model used to create the profiles was assessed under a variety of conditions. The first test involved no added noise or variability, just to demonstrate E-CRAM's performance under an ideal set of conditions. Spatial variability in $K_{2}$ was assessed based upon a spatial standard deviation of the retrieved spectral backscatter ratio. This variability was in fact assessed as a function of $1 / K_{1}$, for consistency with the convention in the existing CRAM aerosol models describing the ratios as 532 to $1064 \mathrm{~nm}$.

The minimization in Fig. 1 is very clear for the ideal case, correctly indicating the appropriate choice of $1 / K_{1}$ of 1.5 , consistent with the parameters used to generate the trial profiles. Obviously, to operate routinely on real data, E-CRAM must be able to find appropriate solutions in the presence of channel noise, as well as in situations in which the assumption of spatially invariant aerosol extinction, backscatter, and lidar spectral ratios does not strictly hold.

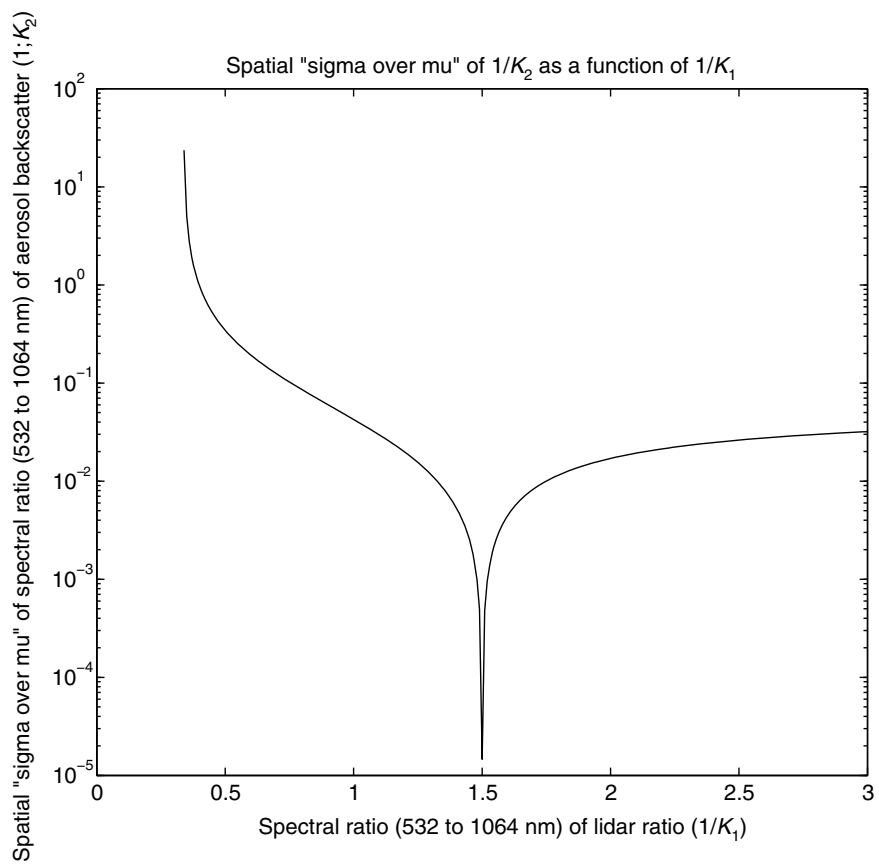

Fig. 1 Minimization plot for the spatial coefficient of variation of $1 / K_{2}$ as a function of $1 / K_{1}$ for the ideal, noiseless case. 


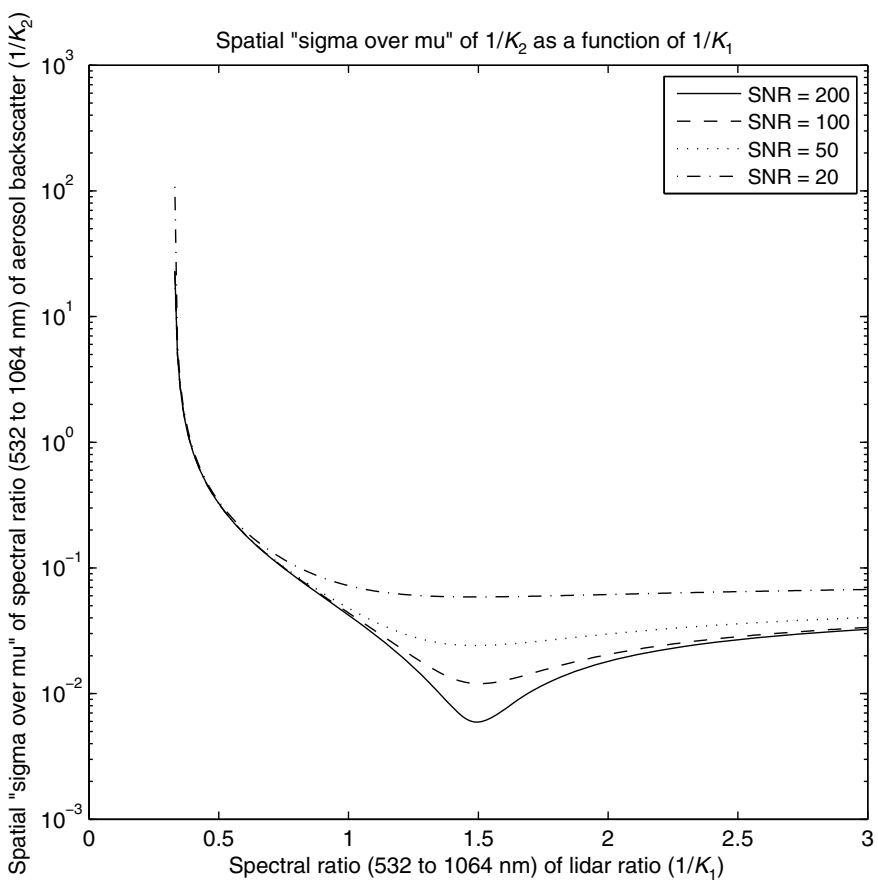

Fig. 2 Minimization plot for the spatial coefficient of variation of $1 / K_{2}$ as a function of $1 / K_{1}$ for a range of SNR values.

The next example illustrates the same minimization, for which the underlying 532- and 1064-nm profiles (aerosol lidar ratios at $532 \mathrm{~nm}$, attenuated backscatter at $1064 \mathrm{~nm}$ ) were generated assuming a range of signal-to-noise ratios (SNRs) between 20 and 200, i.e., with a normally distributed noise term with a standard deviation of 1 /SNR of the mean value in the profile. The effective SNR of the 532-nm lidar ratio and the 1064-nm attenuated backscatter are the principal drivers of the steepness of the minimization. Although less steep in the presence of noise, the minima (Fig. 2) are still fairly clear and indicate a value for $1 / K_{1}$ of exactly 1.5 . Since the technique is meant to operate on some spatially distributed region, having some real distribution of aerosol intensive properties, the minimization acts on the variability over the region as a whole, thereby reducing the effects of random variability within the region to arrive at statistically meaningful aggregate values.

It is also helpful to understand the sensitivity of E-CRAM to calibration errors in HSRL's 1064-nm channel. Another simulation was carried out by effectively varying the 1064-nm calibration by adjusting the 1064-nm attenuated backscatter profile up and down by $10 \%$, the equivalent of a calibration error of the same magnitude. The result (Fig. 3) shows that the error in the value determined for $K_{1}$ was affected by a comparable, and not a disproportionate, margin. Calibration of the 1064-nm channel certainly figures in to the overall error budget in the ultimate determination of the 1064-nm lidar ratio via E-CRAM. Calibration of the 1064-nm channel on the airborne instrument is accomplished in "clean" air, where the aerosol contribution to scattering is negligible, and is estimated to be accurate to within $\sim 5 \%$. ${ }^{13}$ The sensitivity of the technique to bias errors in the 532-nm lidar ratio is almost exactly the same, as shown in Fig. 4. The sensitivity analysis reveals that $10 \%$ bias errors in either of the two parameters to which the technique is sensitive gives rise to almost exactly proportional errors in the coefficient of variation minima for determination of the $K_{1}$ parameter.

\section{Extension Constrained Ratio Aerosol Model-Fit Applied to High Spectral Resolution Lidar Data}

The E-CRAM technique has also already been applied to much of the existing LaRC HSRL dataset. The method has been used to develop and parameterize an independent set of aerosol models for use with CRAM, similar to that of the Cattrell et al. study, but developed on the basis 
McPherson and Reagan: Extension of the constrained ratio approach to aerosol retrievals from...

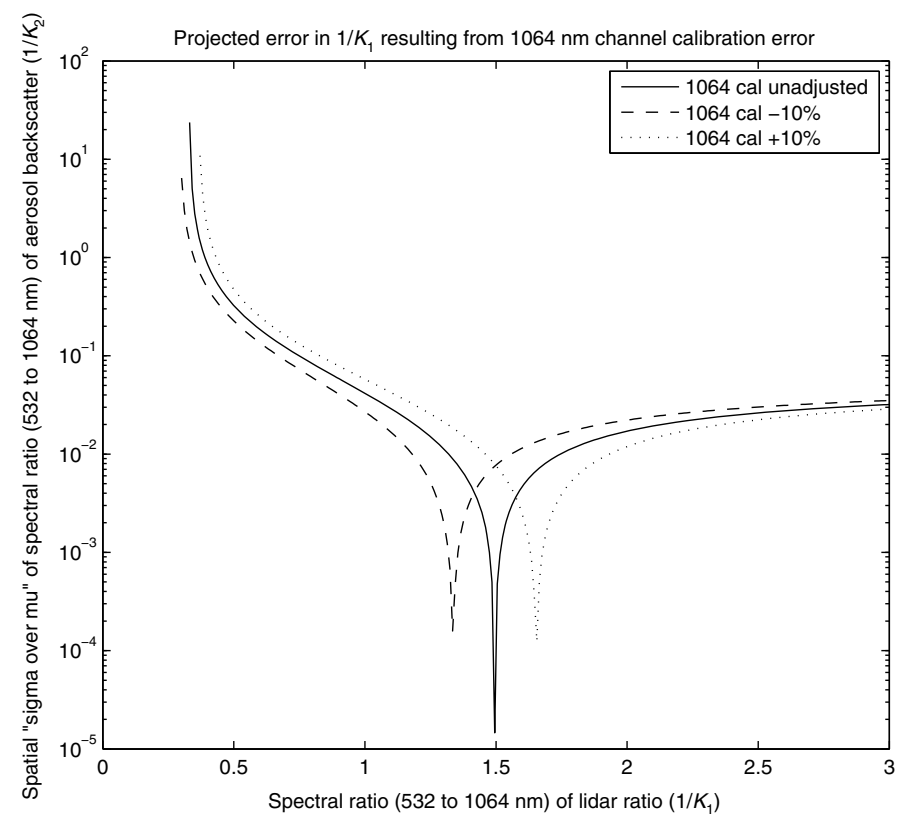

Fig. 3 The resultant shift in the $1 / K_{2}$ spatial coefficient of variation minimization due to $\pm 10 \%$ calibration errors at $1064 \mathrm{~nm}$.

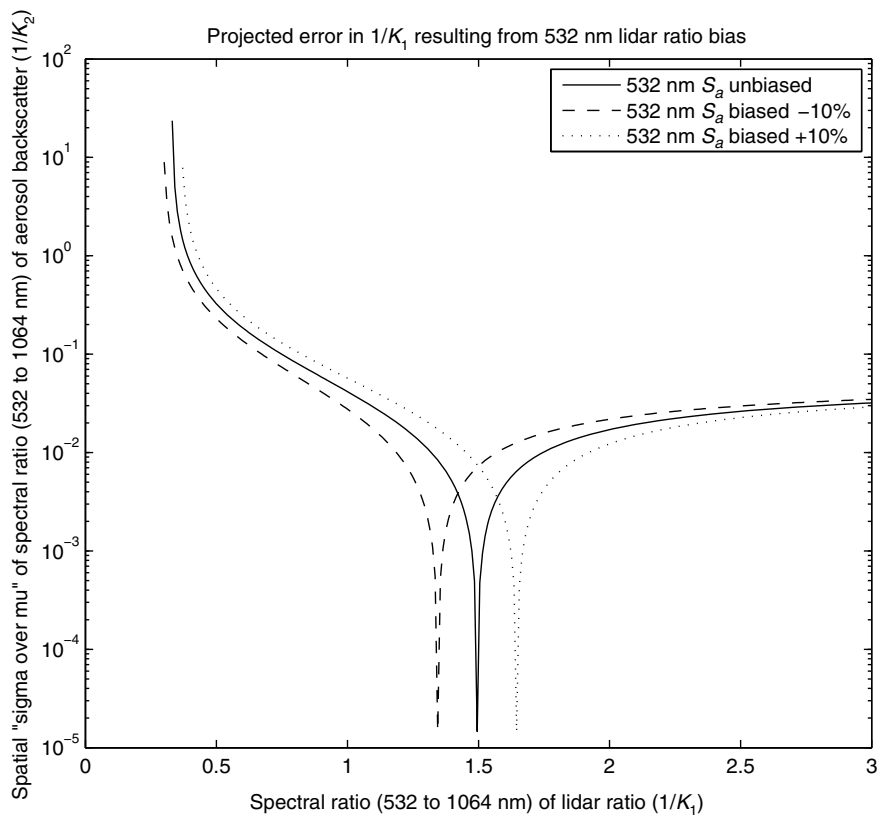

Fig. 4 The resultant shift in the $1 / K_{2}$ spatial coefficient of variation minimization due to $\pm 10 \%$ bias error in the 532-nm lidar ratio.

of large-scale statistical analysis and clustering applied to E-CRAM results from HSRL measurements. ${ }^{23}$ Figure 5 illustrates the geographic distribution of the aerosol measurements by HSRL included in this study. A statistical analysis of a diverse set of aerosol observations from across North America results in a number of distinct aerosol types characterized by spectral ratio parameters that form the basis of a set of aerosol retrieval models. The distribution of 532-nm aerosol optical depth (AODs) observed from the interrogated layers from this study is shown in Fig. 6. Distributions for the various key measured and retrieved aerosol parameters from this study are shown in Fig. 7. As an example of the E-CRAM retrieval process applied in 


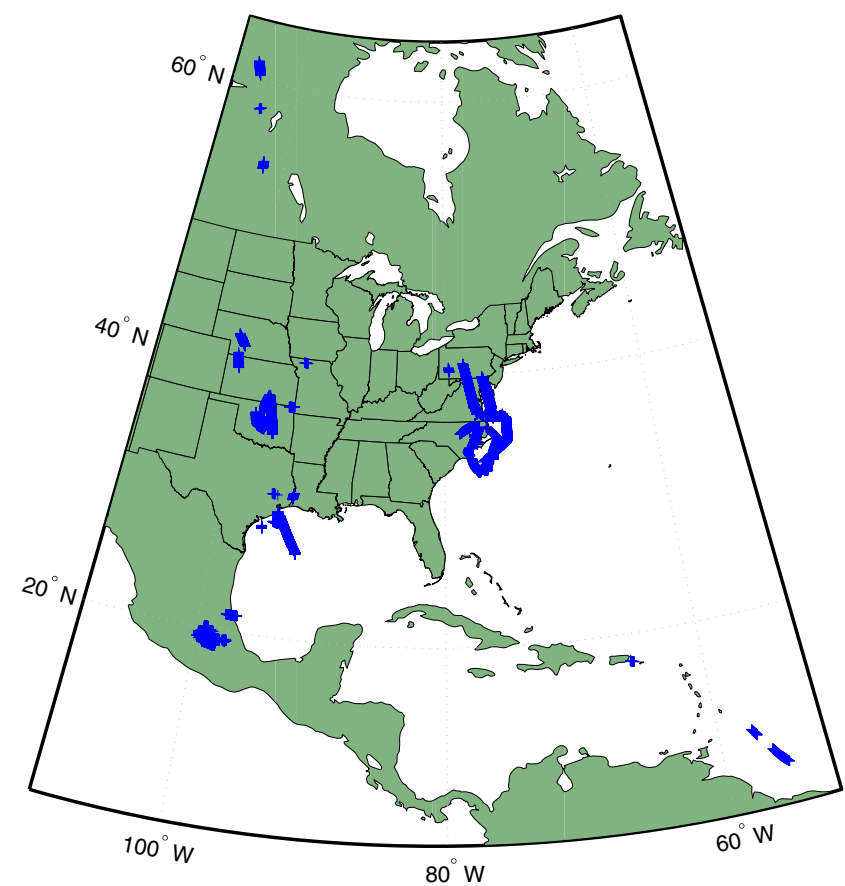

Fig. 5 Geographic distribution of HSRL aerosol layer measurements to which E-CRAM has been applied as part of an ensemble study on aerosol parameterization.

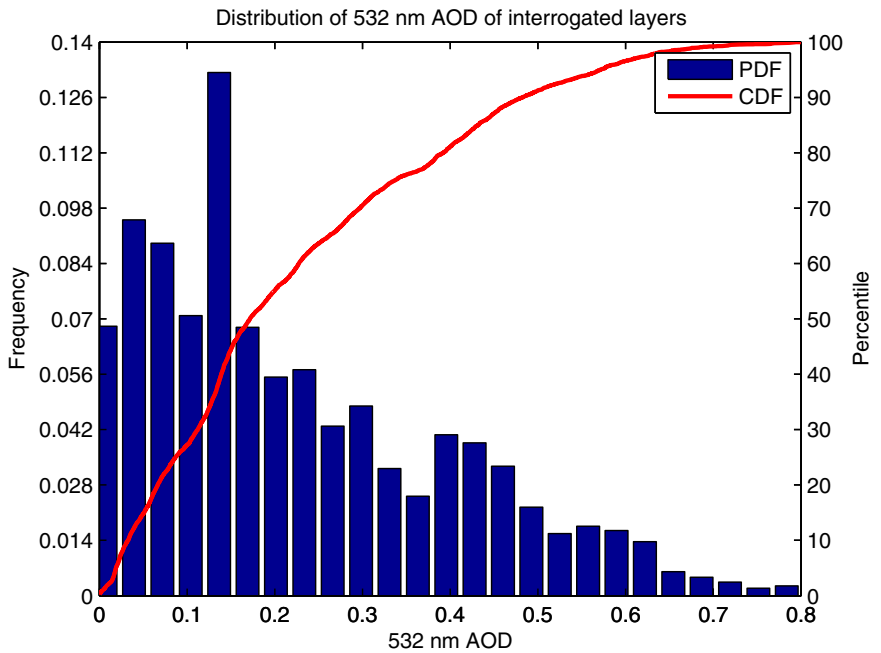

Fig. 6 Distribution of 532-nm AOD as measured by HSRL of interrogated layers comprising the ensemble study.

the study, a track from one of the HSRL's flights is shown in Fig. 8. A region of homogeneous aerosol (in the sense of loading-independent intensive properties) meeting the criteria for application of E-CRAM lies along the red segment of the track shown in Fig. 8. The 532-nm aerosol backscatter and lidar ratio profiles are illustrated in Fig. 9.

By inspection, it is fairly clear that the 532-nm lidar ratio profile indicates that the layer does meet the homogeneity requirements for application of E-CRAM (spatial standard deviation of the 532-nm lidar ratio is constrained to within $15 \%$ of the mean). This is verified numerically in Fig. 10, showing the spatial distribution of the 532-nm lidar ratio throughout the solution region. The standard deviation falls within $\sim 10 \%$ of the mean value. For reference, the CRAM urban/ industrial aerosol model parameter for the 532-nm lidar ratio is bracketed in red. 

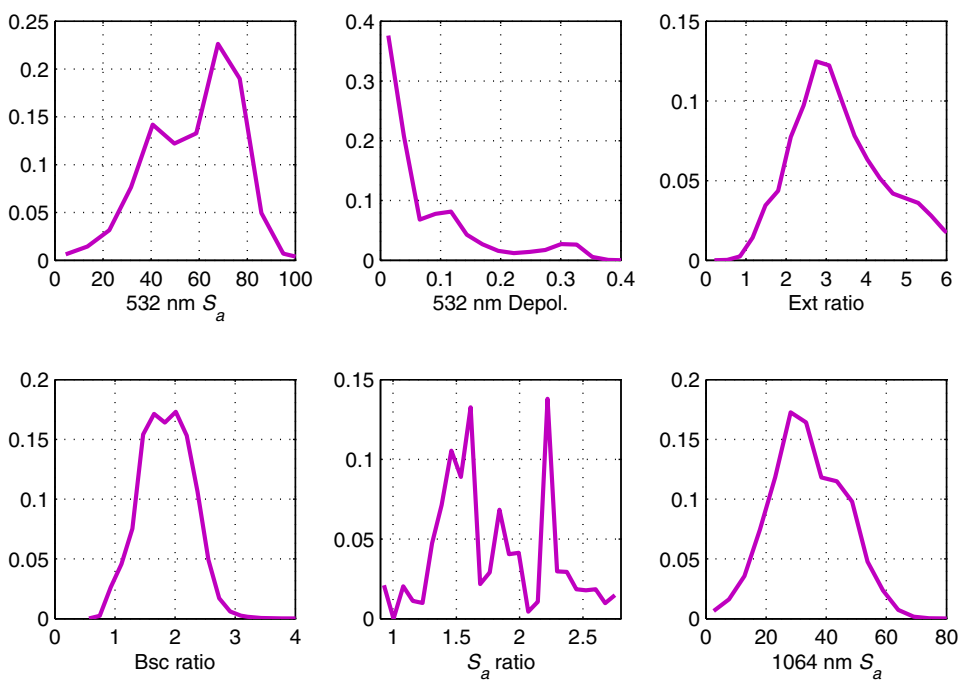

Fig. 7 Distribution of key measured and retrieved aerosol parameters from the ensemble study.

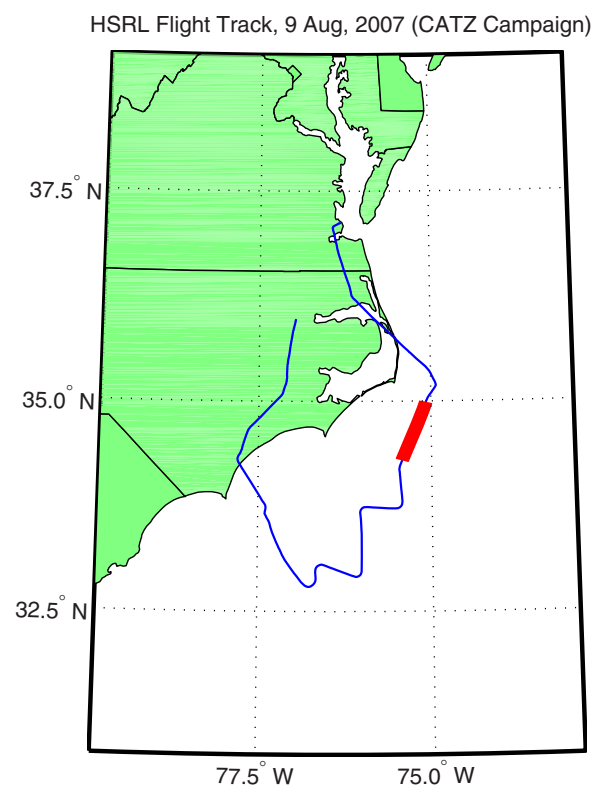

Fig. 8 HSRL flight track from August 9, 2007, off the coast of North Carolina.

Urban-industrial aerosol is a likely choice of model to match this particular layer, given its geographic location and relatively high 532-nm lidar ratio. It is of course also important to establishing the boundary transmittance atop the E-CRAM solution region that there is no overlying aerosol of a different type, or otherwise not meeting the homogeneity requirements of the method. This condition is also clearly satisfied. Applying E-CRAM to this layer, a minimization is obtained as shown in Fig. 11. A minimum in the spatial variability of $1 / K_{2}$ occurs for a value of $1 / K_{1}$ of 1.6. Thus, the 1064-nm lidar ratio is determined everywhere within the retrieval region, relative to the 532-nm lidar ratio, subject to the constraint that all the relevant spectral ratios remain reasonably constant spatially within the solution region. Consequently, as a result of the retrieval process, the aerosol backscatter and extinction are also computed according to the spatially constrained spectral ratio constraints. The resultant spatial distributions on the spectral ratios (532 to $1064 \mathrm{~nm}$ ) of backscatter and extinction are shown in Figs. 12 and 13, respectively.

The one-sigma bounds on the spectral ratios of aerosol backscatter and extinction corresponding to the urban/industrial CRAM model are also shown in Figs. 12 and 13. 

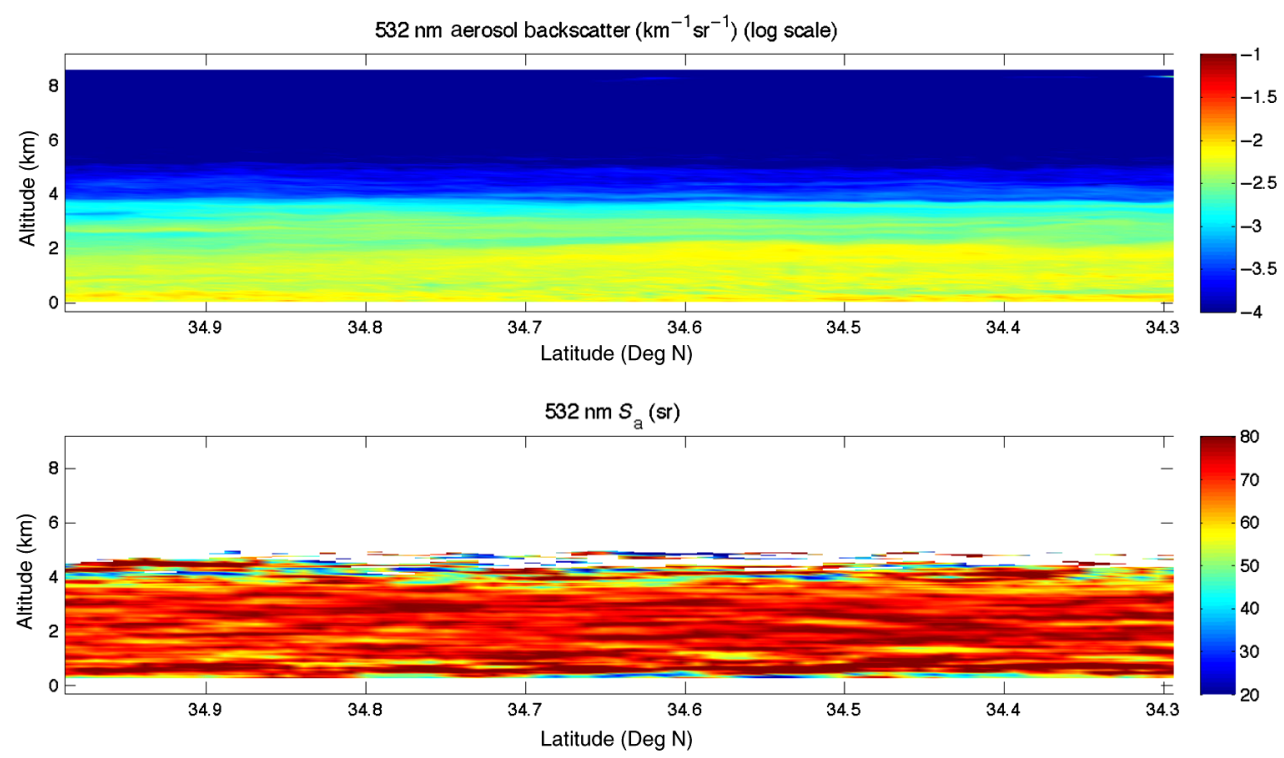

Fig. 9 A 532-nm aerosol backscatter (top) and lidar ratio (bottom) measurements from the LaRC Airborne HSRL instrument over a spatially homogeneous layer of aerosol over coastal North Carolina on August 9, 2007.

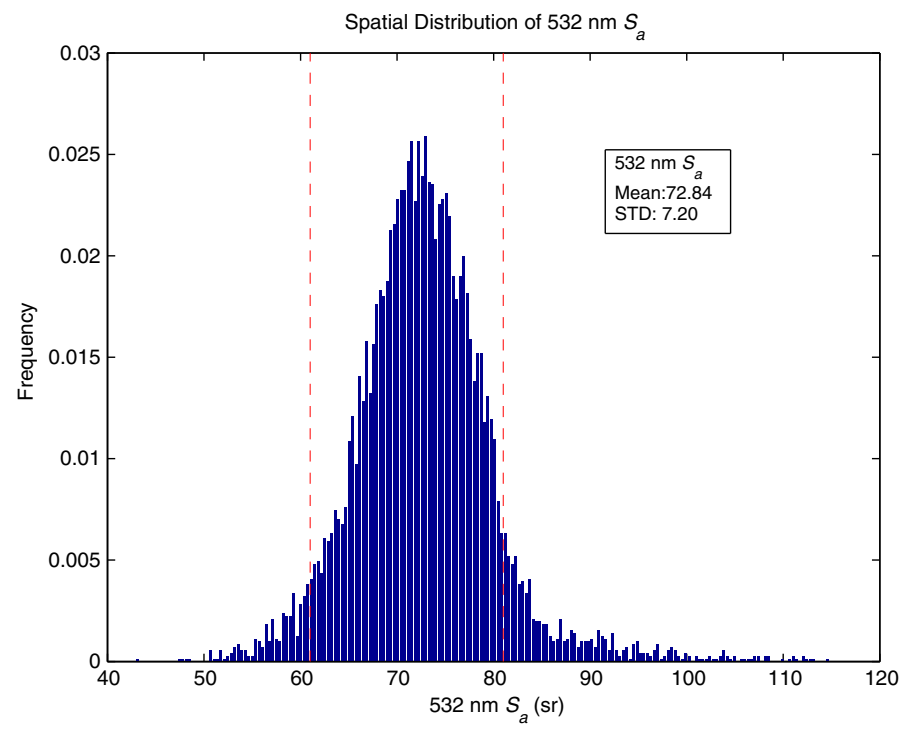

Fig. 10 Spatial distribution of the 532-nm lidar ratio within the aerosol layer identified in Fig. 9. One-sigma limits of CRAM urban/industrial aerosol model are shown in red for reference.

This E-CRAM retrieval suggests that this aerosol layer is fairly consistent with the existing urban/industrial aerosol model in terms of the 532-nm lidar ratio, as well as the spectral ratio of extinction. The mean of the spatial distribution on the retrieved spectral ratio of aerosol backscatter falls somewhat outside the one-sigma bounds of the model, however, which might suggest some adjustment of the model based on additional similar observations. This result is important, in that it demonstrates means by which spectral ratio parameters related to the intrinsic aerosol properties can be independently retrieved via the E-CRAM method applied to HSRL observations with no additional assumptions apart from some reasonable degree of spatial homogeneity in the observed aerosol.

With the abundance of available HSRL data, it is possible to construct an independent aerosol modeling database useful for CRAM-like retrievals from dual-wavelength elastic-scatter instruments. Critically, the E-CRAM method represents one approach to validate and modify existing 


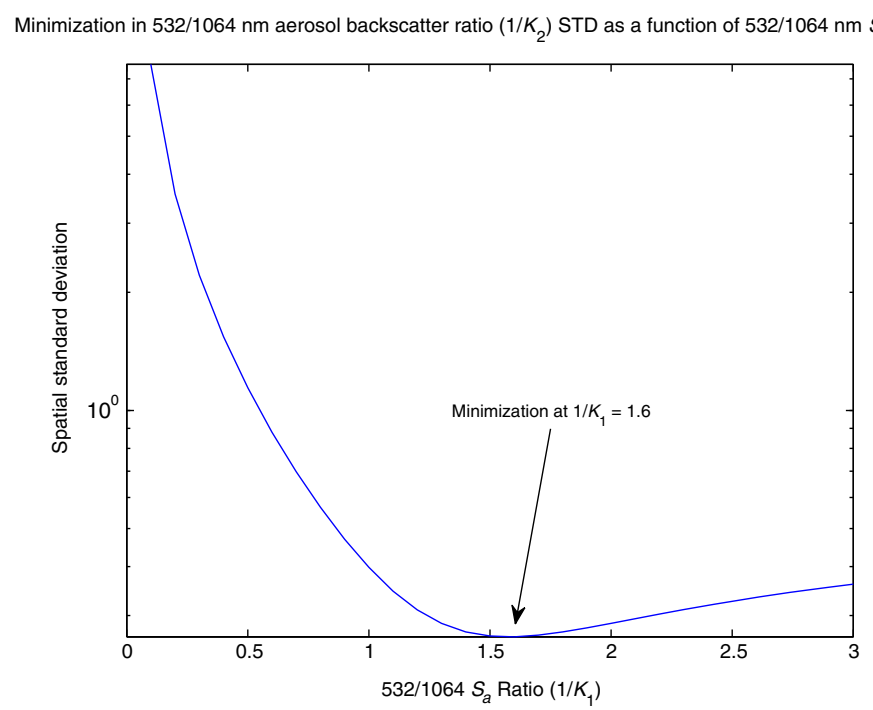

Fig. 11 Result of E-CRAM applied to the region shown in Fig. 9. Minimization in the spatial coefficient of variation of $1 / K_{2}$ occurs for a value of $1 / K_{1}$ of 1.6 .

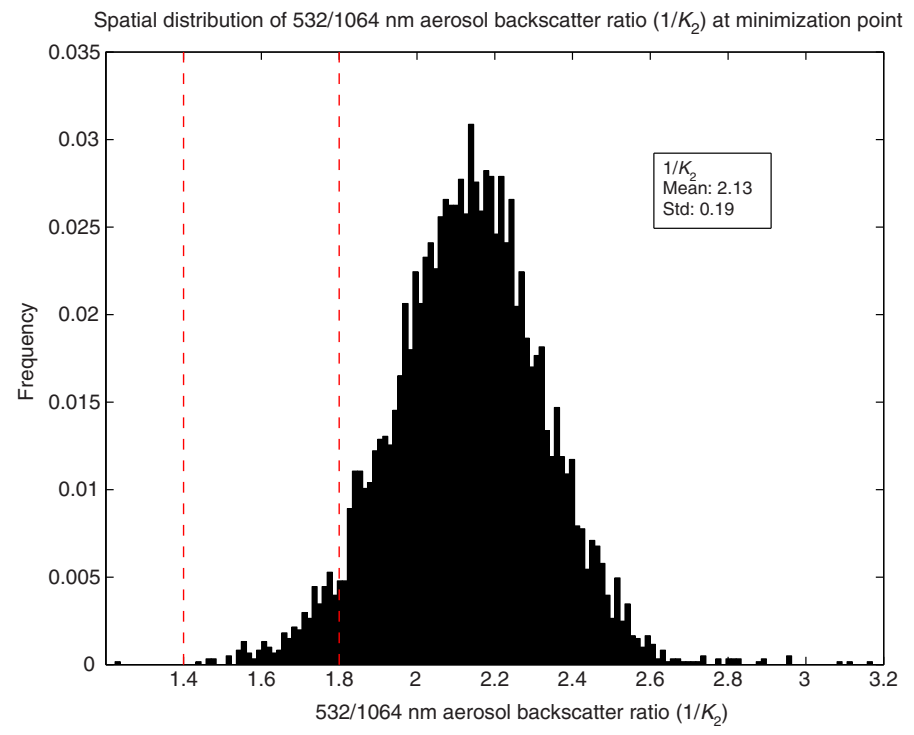

Fig. 12 Final spatial distribution of the spectral ratio (532 to $1064 \mathrm{~nm}$ ) of aerosol backscatter based on the E-CRAM retrieval. The corresponding CRAM model parameter (one-sigma bound) for urban/industrial aerosol is bracketed in red (for reference).

aerosol models based on an entirely different approach and instrument suite (HSRL, as opposed to AERONET observations).

To expand upon the E-CRAM dataset, an automatic layer identification scheme was devised, which incorporated a considerably larger set of data than the original analysis, the spatial distribution of which is shown in Fig. 14. The automated layer identification process began with an existing collection of aerosol layers identified and classified in the same HSRL dataset. ${ }^{24}$ Contiguous layers so identified were down-selected to identify E-CRAM candidate layers satisfying certain criteria, those having maximum spatial extent of 20 min temporally and $1 \mathrm{~km}$ vertically, with a minimum layer thickness of $300 \mathrm{~m}$ vertically and satisfying the E-CRAM 15\% lidar ratio variability constraint, with no substantial overlying aerosol loading $(<0.1532-\mathrm{nm}$ AOD). These criteria identified 1695 candidate layers suitable for E-CRAM retrievals, having ensemble statistics as shown in Fig. 15. 
McPherson and Reagan: Extension of the constrained ratio approach to aerosol retrievals from...

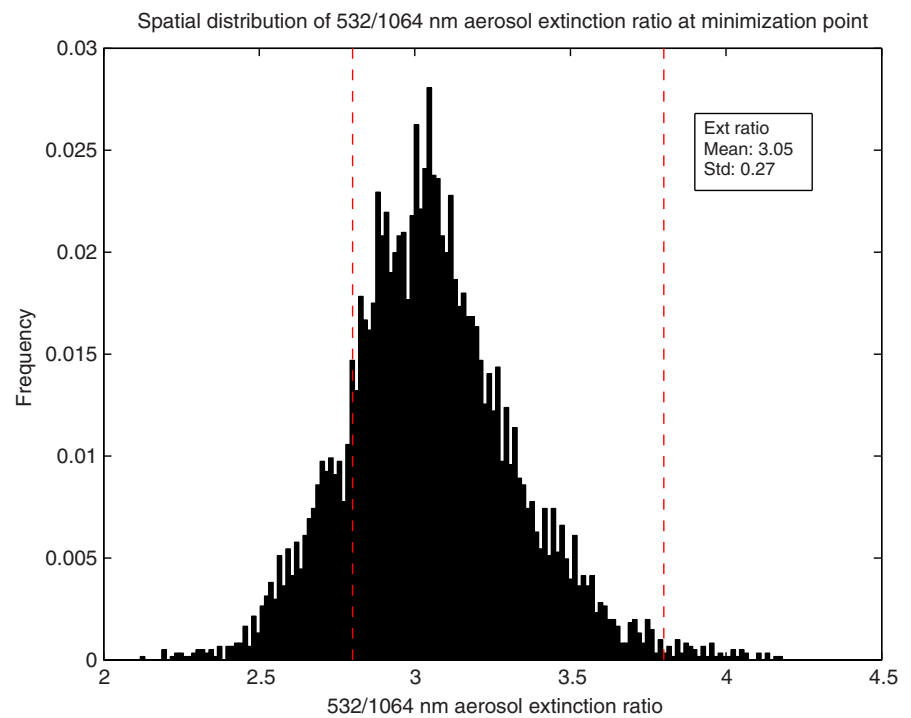

Fig. 13 Final spatial distribution of the spectral ratio (532 to $1064 \mathrm{~nm}$ ) of aerosol extinction based on the E-CRAM retrieval. The corresponding CRAM model parameter (one-sigma bound) for urban/industrial aerosol is bracketed in red (for reference).

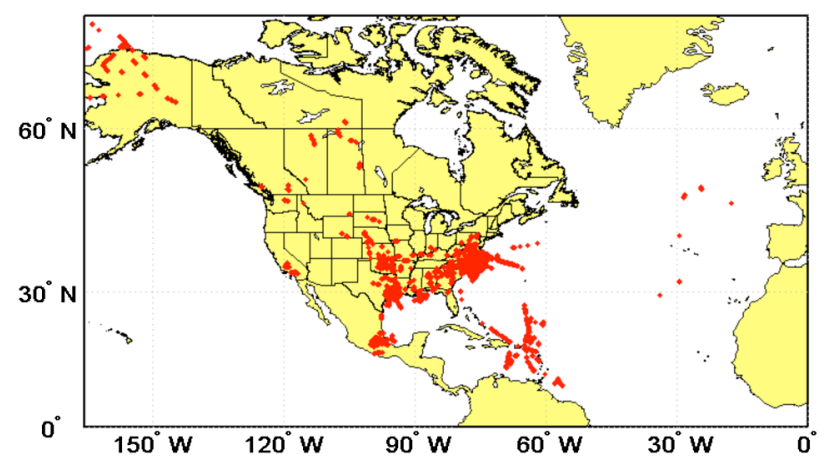

Fig. 14 Geographic distribution of HSRL dataset incorporated in the automated layer identification process.
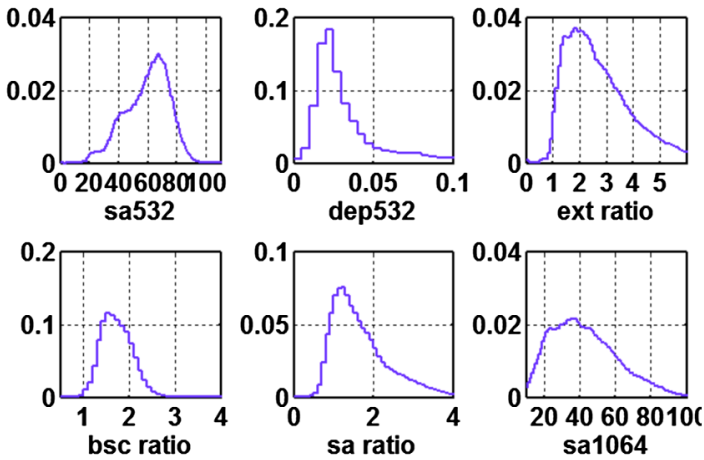

Fig. 15 Ensemble parameter distributions from E-CRAM retrievals from automated layer identification scheme. 


\section{Conclusions}

Herein, we have formulated and demonstrated a method for combined 532-nm HSRL and 1064$\mathrm{nm}$ elastic-scatter lidar retrievals that facilitates the determination of the full aerosol scattering solution at $1064 \mathrm{~nm}$ subject only to fairly minor constraints on spatial homogeneity of the microphysical properties of the observed aerosol. With 532-nm lidar ratio measurements via HSRL available instrumentally to within $10 \%$ and 1064-nm attenuated backscatter available from the instrument theoretically to within $5 \%$, it would not be unreasonable theoretically to expect to be able to determine the 1064-nm lidar ratio to within $10 \%$ to $12 \%$ for aerosol layers meeting the homogeneity constraints imposed by the technique, as well as to determine the 1064-nm aerosol backscatter and extinction profiles to within some comparable margin. While this approach is powerful in and of itself, its greater application may be in the formulation and refinement of aerosol models as suggested in Sect. 4, which could be used to improve model-based constrained retrievals from currently operating dual-wavelength instruments, especially CALIPSO via techniques like CRAM, thereby enhancing the quality of the aerosol data products and expanding the utility of certain derived spectral parameters.

While we have spent some time in Sec. 4 alluding to the conditions under which CRAM and E-CRAM might obtain different results, it is important to take some time to distinguish between the two methods so as to disentangle their particular assumptions and criteria of applicability. CRAM is a model-based retrieval approach that seeks to mutually constrain aerosol solutions at 532 and $1064 \mathrm{~nm}$ so as to be consistent with distinct aerosol models in terms of spectral ratios of retrieved parameters, i.e., aerosol backscatter and extinction. E-CRAM, on the other hand, seeks to constrain a solution only at $1064 \mathrm{~nm}$ based on an available solution at $532 \mathrm{~nm}$ (achieved, e.g., via HSRL or inelastic-scattering means). Both techniques rely on some amount of spatial homogeneity, implicitly in the case of CRAM, but directly in the case of E-CRAM in which the spatial homogeneity of the optical properties of the aerosol can be assessed via direct assessment of the spatial variability of the 532-nm lidar ratio.

Fundamentally, our objective has not been to illustrate the vast variety of cases in which CRAM fails to obtain a suitable solution, and in which E-CRAM succeeds; the limitations of CRAM are acknowledged and well established. Rather, we seek to present E-CRAM as a companion method (as distinct from a successor) to CRAM. Although CRAM and E-CRAM share much of the same heritage in terms of their constraints on spectral ratio parameters, they are distinct methods, each applicable in slightly different circumstances, though their objectives in terms of solutions are essentially the same. There is a vast repository of data that can benefit substantially from a more thorough application of the traditional CRAM method (the CALIOP lidar is probably the best example), by which the uncertainties on 1064-nm aerosol retrievals can be reduced. E-CRAM is a means by which we propose to augment and diversify the set of aerosol models underpinning the CRAM method. Application of E-CRAM to data from instruments such as HSRL makes such improvements possible, and we believe that we have laid out a clear and compelling framework for how the process might work. This seems to be among the more exciting possible applications for E-CRAM. Alternatively, E-CRAM offers a compelling approach to 1064-nm aerosol retrievals from so-called two-beta, one-alpha systems [i.e., attenuated backscatter (one beta) from one channel, and aerosol backscatter and extinction (one beta and one alpha) from a second channel]. Many such systems are beginning to come online, also three-beta, two-alpha systems, to which E-CRAM could also be applied (possibly even more stably) by imposing simultaneous constraints across two wavelengths, and perhaps also relaxing substantially the requirement on spatial homogeneity.

\section{Acknowledgments}

This work benefitted substantially from collaboration with Ray Rogers, formerly at NASA LaRC, specifically from his development of an automated aerosol layer identification routine from which he was able to develop some ensemble results from accumulated data from the NASA LaRC Airborne HSRL instrument. Much of the work presented in this paper was funded under the author's NASA GSRP fellowship, under NASA contract NNX-07AM11H as well as from NASA contract NASA1-99102. The authors would also like to acknowledge NASA's Airborne HSRL program, from which most of the data presented in this paper were made available. 


\section{References}

1. F. G. Fernald, "Analysis of atmospheric lidar observations: some comments," Appl. Opt. 23(5), 652-653 (1984).

2. F. G. Fernald, B. M. Herman, and J. A. Reagan, "Determination of aerosol height distributions by lidar," J. Appl. Meteorol. 11, 482-489 (1972).

3. J. D. Klett, "Stable analytical inversion solution for processing lidar returns," Appl. Opt. 20(2), 211-220 (1981).

4. J. D. Klett, "Lidar iversion with variable backscatter/extinction ratios," Appl. Opt. 24(11), 1638-1643 (1985).

5. S. A. Young, D. B. Josset, and M. A. Vaughan, "Lidar ratios for dust aerosols derived from retrievals of CALIPSO visible extinction profiles constrained by optical depths from MODIS-Aqua and CALIPSO/CloudSat ocean surface reflectance measurements," in Proc., Int. Symp. on the A-Train Satellite Constellation (2010).

6. J. A. Reagan et al., "Spaceborne lidar aerosol retrieval approaches based on aerosol model constraints," in Proc. 2004 IEEE Int. Geoscience and Remote Sensing Symp. (IGARSS'04) (2004).

7. J. A. Reagan et al., "Lidar aerosol retrievals from ICESat using a model-based constrained ratio approach," in Proc. AMS 12th Conf. on Cloud Physics (2006).

8. B. N. Holben et al., "AERONET- a federated instrument network and data archive for aerosol characterization," Rem. Sens. Env. 66(1), 1-16 (1998).

9. C. Cattrall et al., "Variability of aerosol and spectral lidar and backscatter and extinction ratios of key aerosol types derived from selected aerosol robotic network locations," J. Geophys. Res. 110(D10), D005124 (2005).

10. C. J. McPherson et al., "AERONET, airborne HSRL, and CALIPSO aerosol retrievals compared and combined: a case study," J. Geophys. Res. 115(D4), D00H21 (2010).

11. D. Müller et al., "Aerosol type-dependent lidar ratios observed with Raman lidar," J. Geophys. Res. 112(D16), D16202 (2007).

12. A. Ansmann, M. Riebesell, and C. Weitkamp, "Measurement of atmospheric aerosol extinction profiles with a Raman lidar," Opt. Lett. 15(13), 746-748 (1990).

13. J. W. Hair et al., "Airborne high spectral resolution lidar for profiling aerosol optical properties," Appl. Opt. 47(36), 6734-6752 (2008).

14. C. Warneke et al., "An important contribution to springtime Arctic aerosol from biomass burning in Russia," Geophys. Res. Lett. 43(13), L01801 (2010).

15. K. Knobelspiesse et al., "Simultaneous retrieval of aerosol and cloud properties during the MILAGRO field campaign," Atmos. Chem. Phys. 11, 6245-6263 (2011).

16. L. T. Molina et al., "An overview of the MILAGRO 2006 campaign: Mexico City emissions and their transport and transformation," Atmos. Chem. Phys. 10, 8697-8760 (2010).

17. B. de Foy et al., "Aerosol plume transport and transformation in high spectral resolution lidar measurements and WRF-flexpart simulations during the MILAGRO field campaign," Atmos. Chem. Phys. 11, 3543-3563 (2011).

18. Z. Liu et al., "CALIPSO lidar observations of the optical properties of Saharan dust: a case study of long-range transport," J. Geophys. Res. 113(D7), D008878 (2008).

19. D. D. Parrish et al., "Overview of the second Texas air quality study (TexAQS II) and the Gulf of Mexico atmospheric composition and climate study (GoMACCS)," J. Geophys. Res. 114(D7), D011842 (2009).

20. S. P. Burton et al., "Using airborne high spectral resolution lidar data to evaluate combined active plus passive retrievals of aerosol extinction profiles," J. Geophys. Res. 115, D00H15 (2010).

21. J. A. Reagan et al., "Using combined $532 \mathrm{~nm}$ HSRL and $1064 \mathrm{~nm}$ elastic-scatter lidar observations to verify and update CRAM dual-wavelength aerosol retrieval models," in IEEE Int. Geoscience and Remote Sensing Symp. (IGARSS'08) (2008).

22. Y. Sasano and E. V. Browell, "Light scattering characteristics of various aerosol types derived from multiple wavelength lidar observations," Appl. Opt. 28, 1670-1679 (1989).

23. C. J. McPherson, "Refinement of CALIPSO aerosol retrieval models through analysis of airborne high spectral resolution lidar data," PhD Dissertation, College of Optical Sciences, University of Arizona (2011). 
McPherson and Reagan: Extension of the constrained ratio approach to aerosol retrievals from...

24. S. P. Burton et al., "Aerosol classification using airborne high spectral resolution lidar measurements- methodology and examples," Atmos. Meas. Tech. 5, 73-98 (2012).

Christopher J. McPherson is a senior optical engineer at Arizona Optical Systems, LLC, in Tucson, Arizona, where he works on developing optical metrology techniques for large, aspheric, freeform optics for astronomy, aerospace, and defense applications. His research interests include remote sensing electro-optical instrumentation and calibration, atmospheric lidar, algorithms, and computational imaging for remote sensing.

John A. Reagan is a professor emeritus of electrical and computer engineering and optical sciences at the University of Arizona, where he has worked in teaching and research for more than 40 years. His research interests include remote sensing with emphasis on atmospheric optics, laser radar (lidar), solar radiometry, and optoelectronics. 